\title{
Classical counterparts of quantum attractors in generic dissipative systems
}

\author{
Gabriel G. Carlo, ${ }^{1, *}$ Leonardo Ermann, ${ }^{1}$ Alejandro M. F. Rivas, ${ }^{1}$ María E. Spina, ${ }^{1}$ and Dario Poletti ${ }^{2}$ \\ ${ }^{1}$ Departamento de Física, CNEA, Libertador 8250, (C1429BNP) Buenos Aires, Argentina \\ ${ }^{2}$ Singapore University of Technology and Design, 8 Somapah Road, Singapore 487372, Singapore
}

(Received 7 March 2017; published 5 June 2017)

\begin{abstract}
In the context of dissipative systems, we show that for any quantum chaotic attractor a corresponding classical chaotic attractor can always be found. We provide a general way to locate them, rooted in the structure of the parameter space (which is typically bidimensional, accounting for the forcing strength and dissipation parameters). In cases where an approximate pointlike quantum distribution is found, it can be associated with exceptionally large regular structures. Moreover, supposedly anomalous quantum chaotic behavior can be very well reproduced by the classical dynamics plus Gaussian noise of the size of an effective Planck constant $\hbar_{\text {eff }}$. We give support to our conjectures by means of two paradigmatic examples of quantum chaos and transport theory. In particular, a dissipative driven system becomes fundamental in order to extend their validity to generic cases.
\end{abstract}

DOI: 10.1103/PhysRevE.95.062202

\section{INTRODUCTION}

The study of quantum to classical correspondence in dissipative systems is attracting a lot of attention nowadays. This is related to its relevance for many different fields that range from the theoretical aspects of quantum information [1,2] to applications such as in cold atoms [3-6]. There is a new body of work that asks for a better understanding of the interplay between the classical and quantum properties of dissipation. We can mention developments in reservoir engineering, which have been applied to generate robust quantum states in the presence of decoherence [7], for example. Optomechanics [8] is also revealing as a promising field where our knowledge of the many intricate features of the route to chaos, so deeply investigated in classical systems, needs to be extended to the quantum arena. Very recently, interesting properties of many-body systems have been elucidated $[9,10]$, and the corresponding classical equations could bring a different perspective when analyzed in terms of the signatures they imprint on the original quantum systems. A rocked open BoseHubbard dimer has shown a nontrivial connection between the interactions and bifurcations in the mean field dynamics. Subsequently, it is of the utmost importance to clarify any details that could be controversial.

Along this line, attention has been directed towards the effects of the monitoring (coupling) details on the emergence or suppression of chaos [11]. Moreover, an apparent paradox regarding regular quantum behavior corresponding to a classical chaotic one in an optomechanical system has been nicely explained owing to studies undertaken from the correspondence perspective [12]. Finally, it was recently claimed that quantum chaotic attractors (i.e., complex quantum equilibrium states typically associated with classical chaotic attractors) with no classical counterpart exist in the open dissipative quantum Duffing system [13].

In order to shed light on some of these features, we study two paradigmatic systems: a dissipative modified kicked rotator map (DMKRM) which has been very fruitful in directed transport theory [14], and a dissipative periodically

*carlo@tandar.cnea.gov.ar driven dynamical system (DPDDS) that has applications in isomerization reactions [15] and which is fundamental to support the generic nature of our ideas. We concentrate on the case of a small but finite value of the effective Planck constant $\hbar_{\text {eff }}$. We have found that by suitably exploring the parameter space of these systems we are always able to find a classical chaotic attractor corresponding to any quantum chaotic one, even when the classical dynamics is regular (we propose a general way to do it). For the exceptional cases where no chaotic region is near the regular one in this space, the quantum limiting distributions also become regular (within quantum uncertainty). The addition of Gaussian noise of size $\hbar_{\text {eff }}$ to the classical equations provides us with the main features of the quantum evolution. The study of a generic system, beyond the kicked ones, has been fundamental to extend the validity of these conjectures [16]

We have organized our paper as follows: In Sec. II, we describe the models, including the way extra Gaussian noise is added to the classical versions in order to find the quantum behavior. The methods to integrate the equations are also explained. In Sec. III, we explore several key values of the parameters in order to show our main point, i.e., that a classical analog can always be found for quantum chaotic attractors. Also, we explain the exceptional cases where pointlike quantum distributions exist. In Sec. IV, we conclude.

\section{MODELS AND CALCULATION METHODS}

\section{A. Dissipative modified kicked rotator map}

The first model we consider is a particle moving in one dimension $[x \in(-\infty,+\infty)]$, periodically kicked by the potential

$$
V(x, t)=k\left[\cos (x)+\frac{a}{2} \cos (2 x+\phi)\right] \sum_{m=-\infty}^{+\infty} \delta(t-m \tau),
$$

where $k$ is the strength of each kick and $\tau$ is the kicking period. When adding dissipation, we obtain the following map [14],

$$
\begin{aligned}
& \bar{n}=\gamma n+k[\sin (x)+a \sin (2 x+\phi)], \\
& \bar{x}=x+\tau \bar{n} .
\end{aligned}
$$


Here, $n$ is the momentum variable conjugated to $x$ and $\gamma$ $(0 \leqslant \gamma \leqslant 1)$ is the dissipation parameter. A conservative limit is reached at $\gamma=1$, whereas the value $\gamma=0$ gives maximum damping. In order to simplify the parametric dependence, it is usual to introduce a rescaled momentum variable $p=\tau n$ and the quantity $K=k \tau$. This is a paradigmatic model in directed transport. As such, it shows a current that emerges as a consequence of breaking the spatial and temporal symmetries (when $a \neq 0$ with $\phi \neq m \pi$, and $\gamma \neq 1$ ). It is worth mentioning that we take $a=0.5$ and $\phi=\pi / 2$ for this work.

Some of us have conjectured [16] that the main effects of quantum fluctuations are similar to those of Gaussian fluctuations of the order of $\hbar_{\text {eff }}$ that are induced in the classical map (we define the effective Planck constant $\hbar_{\text {eff }}$ below). In order to introduce them we modify the first line of Eq. (2) by simply adding $\xi$ (i.e., the random fluctuations). We have chosen to leave no free parameters in order to test the behavior of our conjecture in this situation, so we fix $\left\langle\xi^{2}\right\rangle=\hbar_{\text {eff }}$, having zero mean. However, the exact coincidence of the size of the fluctuations with $\hbar_{\text {eff }}$ is not essential for it to be valid.

The quantum model (without noise) is obtained via $x \rightarrow \hat{x}$, $n \rightarrow \hat{n}=-i(d / d x)(\hbar=1)$. Since $[\hat{x}, \hat{p}]=i \tau$ (where $\hat{p}=$ $\tau \hat{n})$, the effective Planck constant is $\hbar_{\text {eff }}=\tau$. The classical limit corresponds to $\hbar_{\text {eff }} \rightarrow 0$, while $K=\hbar_{\text {eff }} k$ remains constant. Dissipation at the quantum level is introduced by means of the master equation [17] for the density operator $\hat{\rho}$ of the system,

$$
\dot{\hat{\rho}}=-i\left[\hat{H}_{s}, \hat{\rho}\right]-\frac{1}{2} \sum_{\mu=1}^{2}\left\{\hat{L}_{\mu}^{\dagger} \hat{L}_{\mu}, \hat{\rho}\right\}+\sum_{\mu=1}^{2} \hat{L}_{\mu} \hat{\rho} \hat{L}_{\mu}^{\dagger} \equiv \Lambda \rho .
$$

Here, $\hat{H}_{s}=\hat{n}^{2} / 2+V(\hat{x}, t)$ is the system Hamiltonian, $\{$,$\} is$ the anticommutator, and $\hat{L}_{\mu}$ are the Lindblad operators given by $[18,19]$

$$
\begin{aligned}
& \hat{L}_{1}=g \sum_{n} \sqrt{n+1}|n\rangle\langle n+1|, \\
& \hat{L}_{2}=g \sum_{n} \sqrt{n+1}|-n\rangle\langle-n-1|,
\end{aligned}
$$

with $n=0,1, \ldots$ and $g=\sqrt{-\ln \gamma}$ to comply with the Ehrenfest theorem.

\section{B. Dissipative periodically driven dynamical system}

In order to give more general support to our claims, we have chosen to study a full dynamical system that can be thought as a particle moving in the continuously driven time periodic potential

$$
V(x, t)=1-\cos (x)-A \cos \left(2 x+\phi_{a}\right)+k \sin (x)[\cos (t)],
$$

where $k$ is the strength of the time periodic forcing. Throughout this paper we take $A=0.5$ and $\phi_{a}=\pi / 2$. The picture is completed by means of a velocity-dependent damping and Gaussian fluctuations that are usually taken as thermal ones, but that in our present study will play the same role as in the previous model, i.e., reproducing the main effects of quantum fluctuations. It is important to point out that the association of this Gaussian noise with thermal fluctuations is purely formal from our point of view, as in fact our main objective is to reproduce quantum behavior. Moreover, this connection can only be made at the classical level since we do not introduce any temperature in the quantum model. Then, we are led to numerically solve the Langevin equation

$$
m \ddot{x}=-\Gamma \dot{x}-V^{\prime}(x, t)+\xi .
$$

As usual, $x$ stands for the spatial coordinate of the particle, $m$ for its mass (we take $m=1$ ), and $\Gamma$ is the amount of dissipation. Again, in a way similar to the DMKRM case, the Gaussian white noise having zero mean $\xi$ is simply asked to satisfy $\left\langle\xi(t) \xi\left(t^{\prime}\right)\right\rangle=\hbar_{\text {eff }} \delta\left(t-t^{\prime}\right)$.

In this system it is interesting to simulate a molecule with two stable isomers that is under the influence of a monochromatic laser field pulse, for which the term $\sin (x)$ represents dipole coupling [15]. But it is also of a general nature, and the results obtained through its study can be directly applied to many different situations, including, for example, many-body systems [9].

Quantum mechanical evolution is performed by means of integrating the corresponding Lindblad equation, which essentially is Eq. (3) now taking into account the new $V(x, t)$. Since this potential (in contrast with the previous kicked system) is a generic time-dependent one, we use a modified split operator method $[15,20]$ to actually perform this calculation. In fact, we compose unitary steps given by the kinetic and potential terms of the Hamiltonian, and other purely dissipative ones. In order to treat these latter ones, we use the same model as above. For the sake of completeness, we explicitly write down the dissipative part of the Lindblad equation for the density matrix of this system, which can be written as a completely positive map $\mathbf{D}_{\alpha}(d t)$ in the operator-sum or Kraus representation

$$
\rho(t+d t)=\mathbf{D}_{(\varepsilon, T)}(d t)[\rho(t)]=C_{0} \rho(t) C_{0}^{\dagger}+C_{1}^{ \pm} \rho(t) C_{1}^{ \pm \dagger},
$$

where

$$
C_{0}=\mathbb{1}-\frac{1}{2} C_{1}^{ \pm \dagger} C_{1}^{ \pm}, \quad C_{1}^{ \pm}=\sum_{j} \sqrt{\varepsilon d t j}\left|p_{ \pm j \mp 1}\right\rangle\left\langle p_{ \pm j}\right|,
$$

can be interpreted as infinitesimal Kraus operators obeying the rule $\sum_{\mu} C_{\mu}^{ \pm \dagger} C_{\mu}^{ \pm}=\mathbb{1}$ to first order in $d t$ [21]. We take $p$ as the momentum variable, conjugated to the $x$ coordinate. In fact, the two different operators denoted by the superscript \pm are associated with the positive and negative values of the $p$ spectrum. It is important to notice that in this case $\varepsilon$ is the system-bath coupling parameter and can be directly associated with the classical friction parameter $\Gamma$. Also, $\Gamma$ is taken differently from $\gamma$ in the DMKRM, since in generic dynamical systems $\Gamma=0$ corresponds to the dissipationless regime, while in kicked systems $\gamma=0$ stands for maximum damping.

\section{ASSOCIATING A CLASSICAL CHAOTIC ATTRACTOR TO EACH QUANTUM CHAOTIC ONE}

\section{A. Dissipative modified kicked rotator map}

The main tools used for our investigation are the Liouville and Husimi [22] limiting distributions of our systems. For 
the DMKRM we have evolved $10^{4}$ random initial conditions in the $p \in[-\pi ; \pi]$ band of the cylindrical phase space in the classical case (unless otherwise mentioned). For the quantum version we use the Husimi distribution of the evolved initial density matrix corresponding to these classical initial conditions. A very simple way to see the chaoticity or simplicity of these sets is by means of the participation ratio $\eta=\left[\sum_{i} P\left(p_{i}\right)^{2}\right]^{-1} / N$. This measure has its origin as a good indicator of the fraction of basis elements that effectively expand the quantum state. It should be mentioned that the participation ratio depends on the nature of the basis. We have chosen the momentum one, which is good for our purposes since it allows us to tell whether or not a distribution is pointlike. Moreover, we have extended this concept to the classical case. The corresponding $\eta$ is calculated by taking a discretized $p$ distribution after 5000 time steps, which we have verified is enough to reach a reasonable convergence. The number of bins is given by the Hilbert space dimension used in the quantum calculations, which in our case is $N=3^{6}$. It is clear that a finer coarse graining would slightly change the classical $\eta$ distributions, but this will not affect their main properties. This is because the distance among points of the simple limit cycles is almost always greater than the chosen bin size. The quantum equilibrium distribution is obtained in a few periods, and we have taken 50 .

We have explored the parameter space of the DMKRM in a relevant region where many regular isoperiodic stable structures (ISSs [16,23-26], originally called periodicity hubs [27]) appear. They are characterized by low values of $\eta$ and can be noticed as the clear areas with sharp borders in Fig. 1(a). When adding a uniform Gaussian noise of size $\hbar_{\text {eff }}=0.019$, the resulting $\eta$ can be observed in Fig. 1(b). If we accept that this is a good measure of the quantum behavior, it is clear that chaoticity is the rule while very few parameter sets correspond to pointlike structures. When noise of the size of $\hbar_{\text {eff }}$ is added, the parameter space suffers a deep transition and just a couple of regions associated with the largest of these ISSs keep their simplicity. This behavior could seem paradoxical, and (for low dissipation values) apparently it is of a strictly quantum nature, implying that purely quantum chaotic attractors could exist without classical counterparts. But, can the quantum behavior be completely disconnected from the classical behavior of the surrounding structures in parameter space in some cases? The explorations are usually done with the aid of a typical bifurcation diagram, but if we see the whole parameter space, everything becomes clearer. For a representative example of an apparently purely quantum chaotic attractor, we take $k=2.6$ and $\gamma=0.7$. In this case, the phase space is dominated by a period three limit cycle which is shown in Fig. 1(c) (we take $q=\bmod x, 2 \pi$ ). If we add noise of size $\hbar_{\text {eff }}=0.019$, a classical chaotic attractor develops, induced precisely by it and displayed in Fig. 1(d) (in this case we have considered $10^{6}$ random initial conditions with $p \in[-\pi ; \pi]$, and accumulated their evolution over the last 100 periods, from a total of 5000). The similarity with the quantum distribution shown in Fig. 1(f) is remarkable. But this is not all: If we explore the parameter space in the orthogonal direction to $\gamma$ (which is the one typically explored in bifurcation diagrams), we notice that classical chaotic regions are very near, as is the case for the overwhelming majority of regular regions
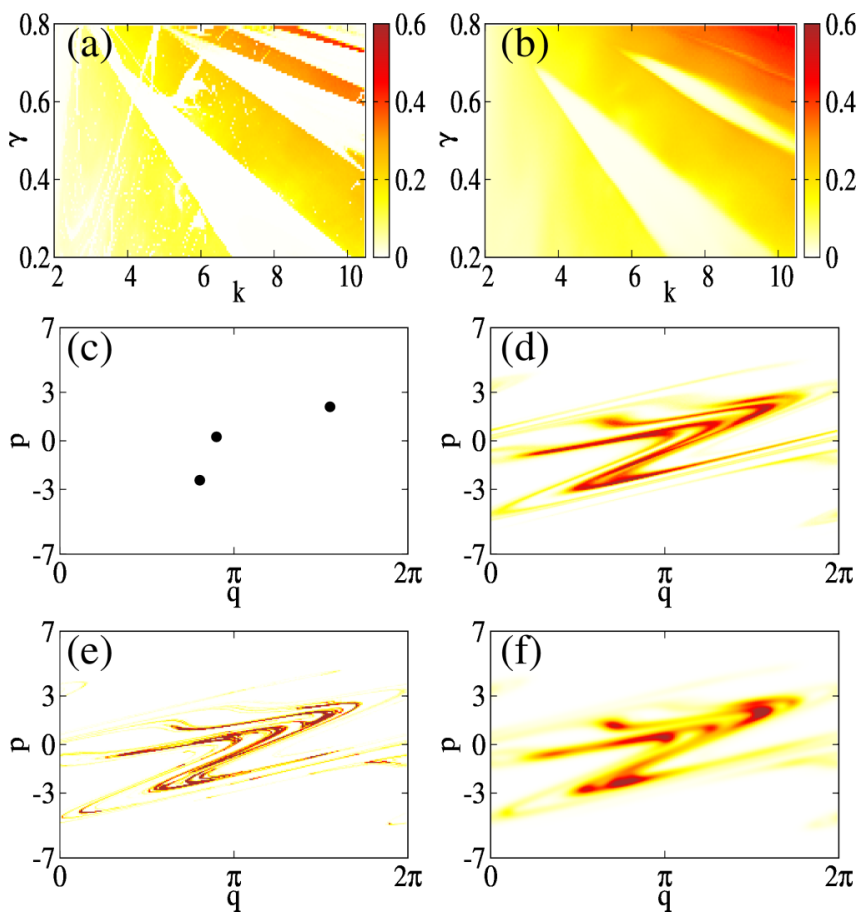

FIG. 1. In (a) we display the participation ratio $\eta$ for the noiseless classical DMKRM. In (b) we show the same quantity when we add Gaussian noise of the size of $\hbar_{\text {eff }}=0.019$. In (c) we show the limit cycle of period three that dominates the phase space at $k=2.6$ and $\gamma=0.7$, while in (d) the classical distribution obtained by adding noise corresponding to $\hbar_{\text {eff }}=0.019$. In (e) we show a classical chaotic attractor (noiseless system) that is found by moving in the $k$ direction ( $\gamma$ fixed) for $k=2.49$ and $\gamma=0.7$. In (f) we display the quantum chaotic attractor found at $k=2.6$ and $\gamma=0.7$, for $\hbar_{\text {eff }}=0.019$.

that are embedded in them. In this case, for $k=2.49$ and $\gamma=0.7$, we have found a classical chaotic attractor that closely resembles the previous chaotic distributions (both classical with noise and quantum) [see Fig. 1(e)]. We define the overlap $O=\iint \mathcal{D}_{1}(x, p) \mathcal{D}_{2}(x, p) d x d p$, where $\mathcal{D}_{1}(x, p)$ and $\mathcal{D}_{2}(x, p)$ are normalized phase space distributions with the same discretization. We have calculated $O$ between the classical distribution with noise of Fig. 1(d) and both the quantum distribution of Fig. 1(f), obtaining $O=0.927$, and the quantum chaotic attractor corresponding to the classical one of Fig. 1(e), obtaining $O=0.905$.

In Fig. 2 we show the details of $\eta$ for different lines along $k$ and $\gamma$, in order to give a more precise explanation of the previous argument. If we move from $k=2.6$, while keeping $\gamma=0.7$, we can see that the chaoticity of distributions grows. In particular, it is the kind of dynamics that dominates for greater $k$ (up to $k \simeq 3.5$, from 4.6 to 6 , and from 7.8 to 9 , approximately), but also for some lower $k$ values [see Fig. 2(a)]. Moreover, if we add Gaussian noise of size $\hbar_{\text {eff }}=0.019$, the intermittency gets washed out, as can be seen by the monotonicity of the (blue) line with circles, with the only exception being the largest regular regions. For comparison purposes, we also show the $\gamma=0.3$ case where, again, the exceptionally large regular region fails to be completely smoothed out by the Gaussian fluctuations, as can be seen from the deep fall in the (red) line with down triangles. 

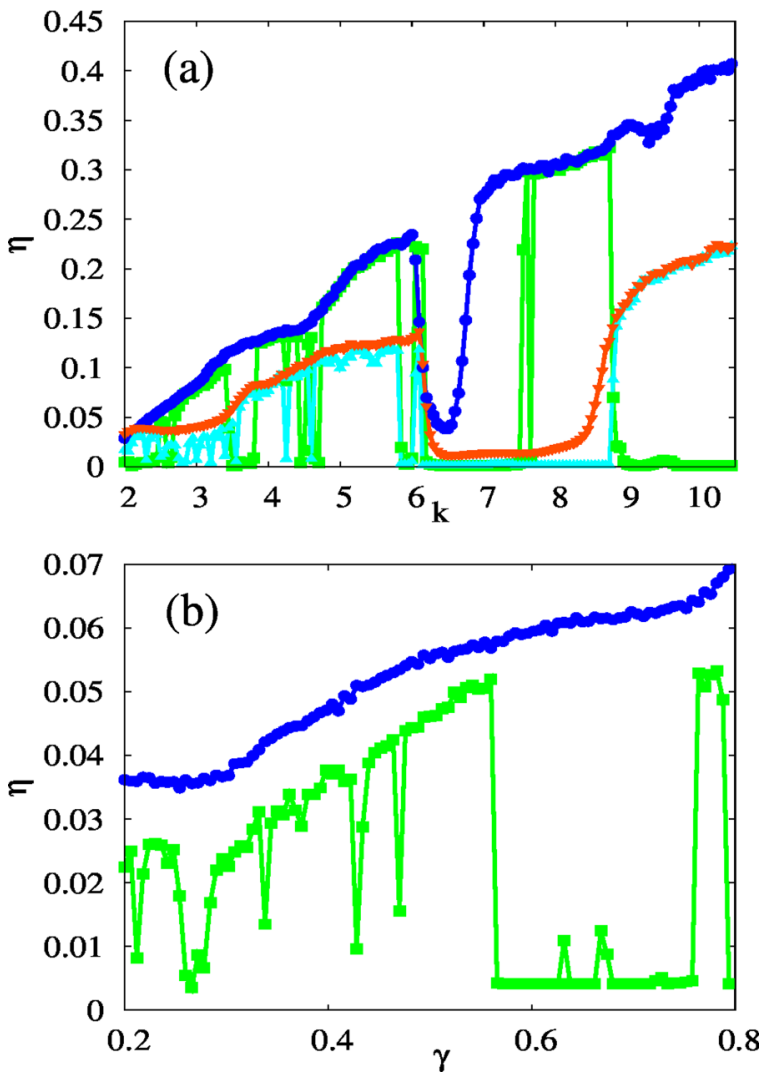

FIG. 2. (a) shows $\eta$ for the DMKRM as a function of $k$. Lines (green) with squares and lines (blue) with circles correspond to $\gamma=$ 0.7. Lines (cyan) with up triangles and lines (red) with down triangles correspond to $\gamma=0.3$. (b) shows $\eta$ as a function of $\gamma$. Lines (green) with squares and lines (blue) with circles correspond to $k=2.6$. Each pair of lines corresponds to the DMKRM without and with Gaussian noise $\left(\hbar_{\mathrm{eff}}=0.019\right)$, respectively.

We now come to Fig. 2(b), where we explore the parameter space in the direction of $\gamma$. If we fix $k=2.6$ and go from $\gamma=0.7$ to lower values, which means increasing the coupling with the environment or equivalently increasing dissipation, it shows that we have a long way to go before arriving at a chaotic region. This is shown by the (green) line with squares. By adding noise we recover the quantum behavior without the need for changing any of the two parameters, as marked by the (blue) line with circles, which follows the largest $\eta$ values associated with the chaotic background.

\section{B. Dissipative periodically driven dynamical system}

We extend the previous results to a generic dissipative system. For that purpose we study the classical and quantum limiting distributions in a stroboscopic surface of a section taken at integer multiples of one period of forcing. For the DPDDS we have evolved 100 random initial conditions in the $p \in[-\pi ; \pi]$ band (we take $p=m \dot{x}$ ) of the cylindrical phase space up to 1500 periods, in the classical case. The Liouville distributions were obtained by accumulating the points of the last 50 periods (unless otherwise noted). This assured a reasonable convergence for these systems that is more numerically demanding to solve than the DMKRM. Again,
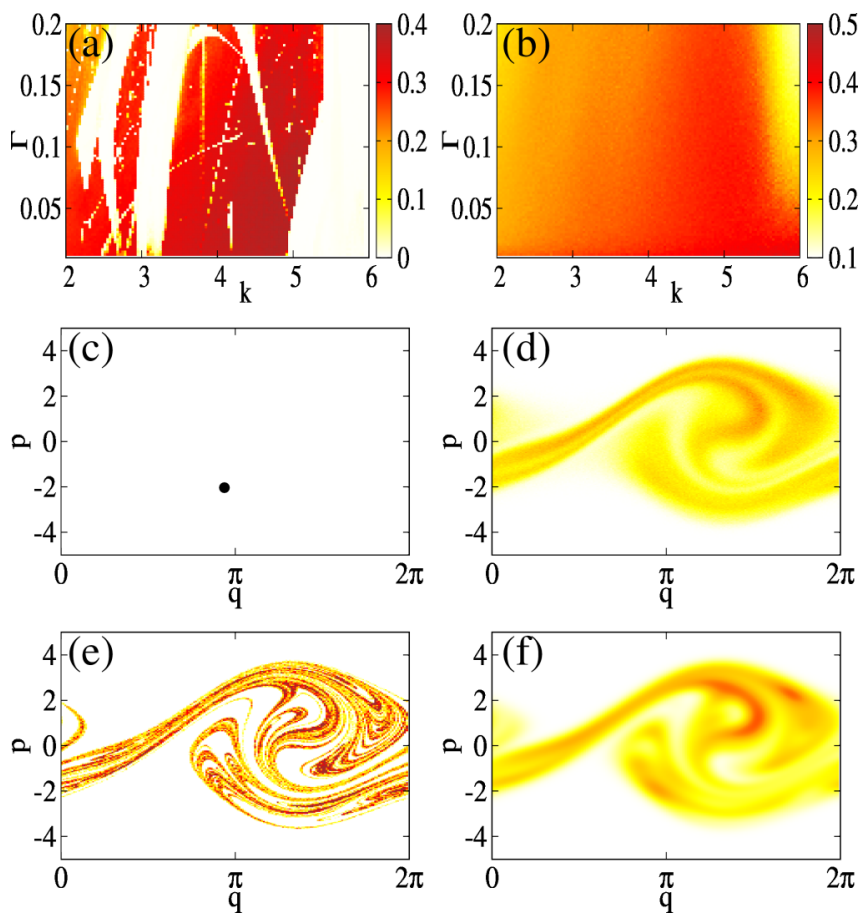

FIG. 3. In (a) we display the participation ratio $\eta$ for the noiseless classical DPDDS. In (b) we show the same quantity when we add Gaussian noise of size $\hbar_{\text {eff }}=0.041$. In (c) we show the limit cycle of period one that dominates the phase space at $k=2.6$ and $\Gamma=0.06$, while in (d) the classical distribution obtained by adding Gaussian noise corresponding to $\hbar_{\text {eff }}=0.041$. In (e) we show a classical chaotic attractor (noiseless system) that is found by moving in the $k$ direction ( $\Gamma$ fixed) for $k=2.75$ and $\Gamma=0.06$. In (f) we display the quantum chaotic attractor found at $k=2.6$ and $\Gamma=0.06$, for $\hbar_{\text {eff }}=0.041$.

for the quantum version we use the Husimi distribution of the evolved initial density matrix corresponding to these classical initial conditions. In this case, the equilibrium distribution is obtained within a few periods (we have taken 50 ).

In Fig. 3(a), it can be seen already that the morphology of the parameter space is quite similar to the one of the DMKRM. In fact, we can identify ISSs all over it, with their typical antennalike features which give them the familiar name of shrimps. We can also find a larger regular structure to the right-hand side that is the only partially surviving one when adding noise of size $\hbar_{\text {eff }}=0.041$ [see Fig. 3(b)]. In Fig. 3(c) we show a period one limit cycle that could be a representative case for a possible purely quantum chaotic attractor located at the low dissipation region, i.e., for $k=2.6$ and $\Gamma=0.06$ (remember that in the DPDDS the dissipation parameter reaches the conservative limit when $\Gamma \rightarrow 0$ ). If we look at Fig. 3(d), showing the classical distribution obtained by adding noise (we have used $10^{4}$ random initial conditions with $p \in[-\pi ; \pi]$ and have accumulated their evolution over the last 500 periods), it becomes clear again that this one is very much similar to that found for the corresponding quantum case, which is shown in Fig. 3(f). Moreover, we are able to find a very similar looking classical chaotic attractor at $k=2.75$ and $\Gamma=0.06$, displayed in Fig. 3(e). The overlap between the classical distribution with noise of Fig. 3(d) and the quantum distribution of Fig. 3(f) is $O=0.983$, and with the quantum 

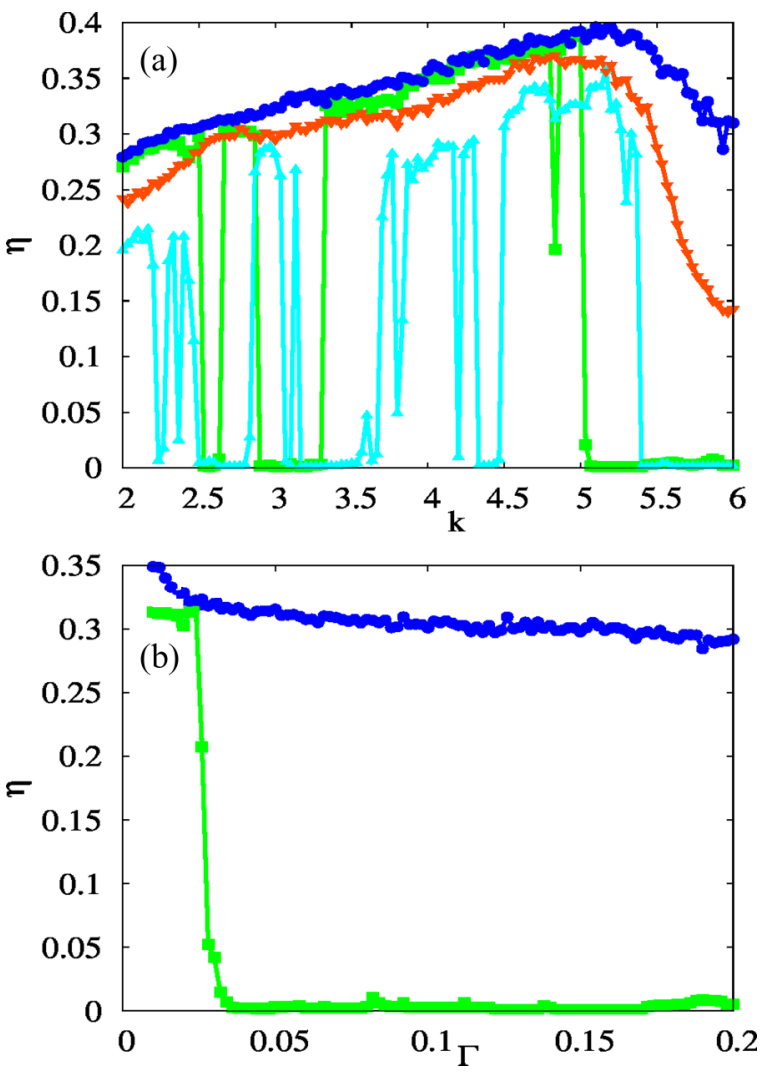

FIG. 4. (a) shows $\eta$ for the DPDDS as a function of $k$. Lines (green) with squares and lines (blue) with circles correspond to $\Gamma=0.06$. Lines (cyan) with up triangles and lines (red) with down triangles correspond to $\Gamma=0.18$. (b) shows $\eta$ as a function of $\Gamma$. Lines (green) with squares and lines (blue) with circles correspond to $k=2.6$. Each pair of lines corresponds to the DPDDS without and with Gaussian noise $\left(\hbar_{\text {eff }}=0.041\right)$, respectively.

chaotic attractor corresponding to the classical one of Fig. 3(e) is $O=0.976$.

The same detailed study of what happens when we explore the parameter space in its two main directions is applicable to this generic system. For example, if we look at Fig. 4(a), we can realize that by going up in the forcing strength $k$ (keeping $\Gamma=0.06)$ will suffice to find a chaotic region beginning approximately at $k=2.7$. This can be seen from the sharp rise in the (green) line with squares. If we add noise, the curve closely follows the largest values corresponding to the chaotic regions, so again we do not need to change any parameter to find the corresponding classical analog of the quantum chaotic attractors. For comparison, we show the $\Gamma=0.18$ case, where the noise only fails to raise the curve at the higher values of $k$ where the largest regular region lies. If we explore in the $\Gamma$ direction, departing from $\Gamma=0.06$ (and fixing $k=2.6$ ), it would take a considerable variation to reach the nearest chaotic region. This can be seen with the aid of the (green) line with squares in Fig. 4(b), which in this case happens at approximately $\Gamma=0.03$. By adding noise we see again how the entire curve rises [see the (blue) line with circles in Fig. 4(b)].

In these two sections we have explored the main directions, the ones corresponding to the system parameters $k$ and $\gamma$.
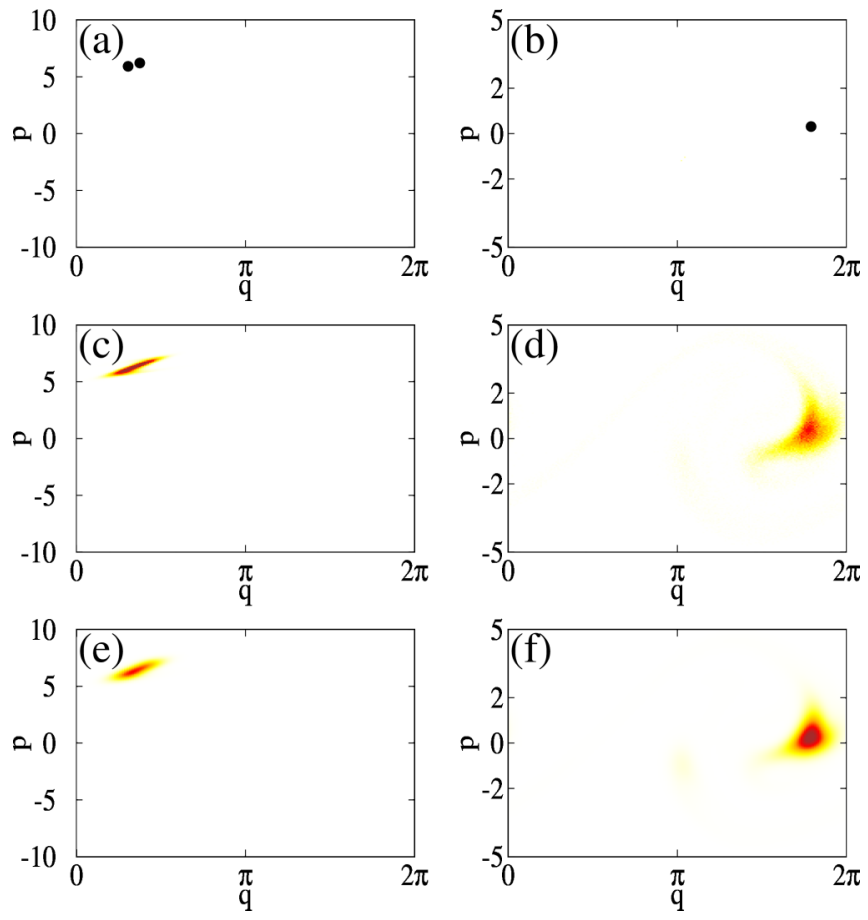

FIG. 5. In the left-hand column the results correspond to the DMKRM and in the right-hand one to the DPDDS. (a) shows the period two limit cycle that dominates the phase space for $k=7.2$ and $\gamma=0.3$, (c) The classical limiting distribution obtained with a Gaussian noise of size $\hbar_{\text {eff }}=0.027$, and (e) the quantum corresponding one. (b) shows the period one limit cycle that dominates the phase space for $k=6.0$ and $\Gamma=0.18$, (d) the classical limiting distribution obtained with a noise of size $\hbar_{\text {eff }}=0.041$, and (f) the quantum corresponding one.

But the general way to find a classical corresponding chaotic attractor for a given quantum one would be to follow the shortest overall variation of both parameters in order to reach the chaotic background. Finally, we have verified this same behavior for several points in parameter space; the ones shown are just representative cases.

\section{Nonchaotic pointlike structures}

The other possibility of finding a purely quantum strange attractor would be to look for the biggest ISSs which can have domains quite far from the chaotic background. But when the chaos is far away, what do the quantum distributions look like? In the left-hand column of Fig. 5 we analyze one representative example for the DMKRM, and in the right-hand one we do the same for the DPDDS (we have verified that the large regular region to which this case belongs extends beyond $k=6$ ).

The period two limit cycle of Fig. 5(a) transforms into an approximately squeezed Gaussian state shown in Fig. 5(c) for the classical DMKRM with Gaussian noise, and in Fig. 5(e) for the quantum DMKRM. It is important to notice that although the quantum distribution is not a point (or two), this behavior is different from the one shown above. These are the simplest, pointlike quantum structures that can be found in this system and do not qualify as purely quantum attractors; they should be associated with simple limit cycles instead (with quantum 
uncertainty, of course). Interestingly, the same happens for the DPDDS, where the period one limit cycle shown in Fig. 5(b) undergoes the same transition to an approximately squeezed Gaussian state both for the classical with Gaussian noise and quantum DPDDS [see Figs. 5(d) and 5(f), respectively].

By looking at Figs. 2(a) and 4(a) we can see that these points of the parameter space correspond to the lowest $\eta$ values for classical systems with noise.

\section{CONCLUSIONS}

Recently, there has been much attention directed towards the properties of the quantum to classical transition in dissipative systems. In particular, the study of the effects of the coupling details on the chaotic behavior [11] and of puzzling results in optomechanics [12] have provided us with very interesting advances. In this context, quantum chaotic attractors with apparently no classical counterpart have been found in the open dissipative quantum Duffing system [13]. On the other hand, despite known discrepancies [16,25] for some limited cases and surviving quantum effects, effective classical maps with Gaussian noise have been proposed as a direct replacement to obtain the main features of quantum dissipative systems. Important consequences have been derived from this identification [26,28].

We have studied two paradigmatic systems, namely, the DMKRM [14], and a generic, continuously driven DPDDS [15] We have found that we can always identify a classical chaotic attractor which corresponds to the quantum one. In general, there are no paradoxes in the quantum to classical correspondence of dissipative systems when we add Gaussian fluctuations to the classical counterparts. When chaotic regions are sufficiently far away from a given regular one, the quantum mechanical attractor is also regular and the distributions become pointlike (with quantum uncertainty). Any quantum attractor can be explained with the help of these two mechanisms. This includes cases where there are coexisting attractors. Given a quantum chaotic attractor as the quantum version of an ISS, the general way of finding the corresponding classical chaotic one consists of varying the parameters along the shortest way in order to reach the nearest chaotic region.

Finally, by analyzing the DPDDS we have been able to extend the validity of our method to find corresponding chaotic attractors and the general correspondence via Gaussian fluctuations to systems where even a semiclassical approximation is hard to obtain. We think that these are generic correspondence properties of dissipative systems [28] that could have many different applications, such as in many-body, optomechanical, and reservoir engineering studies, for example. The influence of coherence and of different noise distributions on the quantum to classical correspondence will be the focus of future studies.

\section{ACKNOWLEDGMENTS}

Support from CONICET under Project No. PIP 112201101 00703 is gratefully acknowledged. D.P. acknowledges support from Singapore Ministry of Education, Singapore Academic Research Fund Tier-I (Project No. SUTDT12015005).
[1] M. A. Nielsen and I. L. Chuang, Quantum Computation and Quantum Information (Cambridge University Press, Cambridge, UK, 2000).

[2] J. Preskill, Lecture Notes for Physics 229: Quantum Information and Computation, http://www.theory.caltech.edu/ people/preskill/ph229/

[3] P. H. Jones, M. Goonasekera, D. R. Meacher, T. Jonckheere, and T. S. Monteiro, Phys. Rev. Lett. 98, 073002 (2007).

[4] T. Salger, S. Kling, T. Hecking, C. Geckeler, L. Morales-Molina, and M. Weitz, Science 326, 1241 (2009).

[5] T. S. Monteiro, P. A. Dando, N. A. C. Hutchings, and M. R. Isherwood, Phys. Rev. Lett. 89, 194102 (2002).

[6] G. G. Carlo, G. Benenti, G. Casati, S. Wimberger, O. Morsch, R. Mannella, and E. Arimondo, Phys. Rev. A 74, 033617 (2006).

[7] D. Kienzler, H.-Y. Lo, B. Keitch, L. de Clercq, F. Leupold, F. Lindenfelser, M. Marinelli, V. Negnevitsky, and J. P. Home, Science 347, 53 (2015).

[8] L. Bakemeier, A. Alvermann, and H. Fehske, Phys. Rev. Lett. 114, 013601 (2015).

[9] M. Hartmann, D. Poletti, M. Ivanchenko, S. Denisov, and P. Hänggi, arXiv:1606.03896.

[10] M. Ivanchenko, E. Kozinov, V. Volokitin, A. Liniov, I. Meyerov, and S. Denisov, arXiv:1612.03444.

[11] J. K. Eastman, J. J. Hope, and A. R. R. Carvalho, arXiv:1604.03494.

[12] G. Wang, Y.-C. Lai, and C. Grebogi, Sci. Rep. 6, 35381 (2016).
[13] B. Pokharel, P. Duggins, M. Misplon, W. Lynn, K. Hallman, D. Andreson, A. Kapulkin, and A. K. Pattanayak, arXiv:1604.02743.

[14] G. G. Carlo, G. Benenti, G. Casati, and D. L. Shepelyansky, Phys. Rev. Lett. 94, 164101 (2005).

[15] G. G. Carlo, L. Ermann, F. Borondo, and R. M. Benito, Phys. Rev. E 83, 011103 (2011).

[16] G. G. Carlo, Phys. Rev. Lett. 108, 210605 (2012).

[17] G. Lindblad, Commun. Math. Phys. 48, 119 (1976).

[18] T. Dittrich and R. Graham, Europhys. Lett. 7, 287 (1988).

[19] R. Graham, Z. Phys. B: Condens. Matter 59, 75 (1985).

[20] J. Javanainen and J. Ruostekoski, J. Phys. A: Math. Gen. 39, L179 (2006).

[21] G. G. Carlo, G. Benenti, G. Casati, and C. Mejía-Monasterio, Phys. Rev. A 69, 062317 (2004).

[22] K. Husimi, Proc. Phys. Math. Soc. Jpn. 22, 264 (1940).

[23] A. Celestino, C. Manchein, H. A. Albuquerque, and M. W. Beims, Phys. Rev. Lett. 106, 234101 (2011).

[24] L. Ermann and G. G. Carlo, Phys. Rev. E 91, 010903(R) (2015).

[25] G. G. Carlo, A. M. F. Rivas, and M. E. Spina, Phys. Rev. E 92, 052907 (2015).

[26] M. W. Beims, M. Schlesinger, C. Manchein, A. Celestino, A. Pernice, and W. T. Strunz, Phys. Rev. E 91, 052908 (2015).

[27] J. A. C. Gallas, Phys. Rev. Lett. 70, 2714 (1993).

[28] G. G. Carlo, L. Ermann, A. M. F. Rivas, and M. E. Spina, Phys. Rev. E 93, 042133 (2016). 